\title{
Study of Metabolite Profiles in Winter Wheat Cultivars Induced by Fusarium Infection
}

\author{
K. Stuper-Szablewska ${ }^{1}$, D. Kurasiak-Popowska ${ }^{2 *}$, \\ J. NAWracaŁA ${ }^{2}$ and J. PERKOWSKI ${ }^{1}$ \\ ${ }^{1}$ Department of Chemistry, Faculty of Wood Technology, Poznan University of Life Sciences, \\ 60-625 Poznan, Poland \\ ${ }^{2}$ Department of Genetics and Plant Breeding, Faculty of Agronomy and \\ Bioengineering, Poznan University of Life Sciences, 60-632 Poznan, Poland
}

(Received 30 October 2015; Accepted 22 April 2016;

Communicated by T. Harangozó)

The aim of this study was to determine the profile of fungal metabolites and antioxidant substances from plants on a representative population of wheat cultivars grown in Poland. Tests were conducted on grain of 23 wheat cultivars in three experimental variants: natural infection (control), chemical crop protection, and artificial inoculation with the fungi F. graminearum and F. culmorum. Grains were analysed in terms of infection rate, mycotoxin, ergosterol, free and bound phenolic acid content, as well as antioxidant capacity. Calculated Fusarium head blight index (FHB index) ranged from $0 \%$ to $24 \%$ in the control, from $0 \%$ to $95 \%$ after inoculation and from 0 to $3 \%$ following chemical crop protection treatments. The highest concentration of ergosterol was detected in seeds from inoculated plots, with a mean value of $26.69 \mathrm{mg} / \mathrm{kg}$. The highest mean concentration calculated for total toxin content was recorded after inoculation $(7.833 \mathrm{mg} / \mathrm{kg})$. The mean value for ferulic acid for inoculated samples resulted $2574 \mathrm{mg} / \mathrm{kg}$, while for chemically protected samples 1158 $\mathrm{mg} / \mathrm{kg}$ was obtained.

Based on of discriminant analysis genotypes can be separated for inoculation $100 \%$, for chemical crop protection 95\%, and for natural infection 90\%. Amongst 12 analysed phenolic acids the highest discrimination power was found for gallic acid, 4-hydroxybenzoic acid and vanillic acid.

Keywords: antioxidants, F. graminearum, F. culmorum, mycotoxins, phenolic acids

\section{Introduction}

Fusarium head blight in cereals is a disease caused by fungi from the genus Fusarium. In Poland, fusarium head blight in wheat is caused mainly by F. culmorum, F. graminearum and $F$. avenaceum (Bottalico 1998). Fungi causing fusarium head blight (FHB) produce numerous metabolites called mycotoxins. The most important and most frequent mycotoxins found in Polish cereal grain are trichothecenes: primarily deoxynivalenol (DON) and nivalenol (NIV) (Bottalico 1998; Perkowski 1999). Since processes of mycotoxin

\footnotetext{
*Corresponding author; E-mail: popowska@up.poznan.pl
} 
inactivation and elimination are costly, time- and labour-consuming, it is most desirable to focus on preventing their formation. It comprises three stages: the first is selection of appropriate cultivars, the second includes cultivation factors, and the third is related to proper grain storage. The most effective methods to reduce losses caused by fusarium head blight in wheat are focusing on application of crop rotation as well as chemical and biological control of disease. Colla et al. (2012) suggest that current EU regulation can lead to further and significant reductions of the available crop protection portfolio, resulting in the loss of active ingredients, having additional impact on the registration of novel products. In this situation the most desirable approach is to grow cultivars resistant to Fusarium, characterised by low DON accumulation in grain (Bartók and Lamper 2003; Chrpová et al. 2010). The resistance to fusarium head blight is influenced by many factors, such as climatic conditions and defence mechanisms in plants. Schaafsma and Hooker (2007) stated, that environment effects accounted for $48 \%$ of the variation in deoxynivalenol (DON) across all fields, followed by variety $(27 \%)$, and previous crop (14 to $28 \%$ ). Additionally, mycotoxin contamination can be divided into that occurring in the developing crop and after maturation which correspond approximately to pre- and post-harvest, respectively (Paterson and Lima 2010).

Head infection, yield, yield components and the percentage of Fusarium-infected grains were determined in many studies connected with FHB (Homdork et al. 2000). With the development of chemical methods, the assessment of plant resistance to fusarium head blight started to be supplemented with chemical analyses, which facilitated the determination of mycotoxin concentrations in grain (Kubo et al. 2014).

Because FHB symptoms and Fusarium trichothecenes levels are not always correlated, breeding for FHB-resistant wheat varieties with lower grain mycotoxin levels is not straightforward and presents some difficulties. This is due to the fact that the mechanisms of plant resistance may also be influenced by other factors such as phenolic acids and other substances with antioxidant properties (Boutigny et al. 2008). In infested cells of plant tissues, the enzymes of biosynthesis of phenolic compounds are activated. In the period preceding pathogen sporulation, the nucleic acid metabolism of the host also changes. The formed phenolic compounds accumulate around the sites of pathogen penetration. As a consequence this process leads to parasite toxication and to necrosis of infested tissues. It is a typical defense response of plants to a pathogen infection.

For this reason it was decided to investigate contents of metabolites, not only fungal, but also those formed in plants as a result of the action of different stressors. In order to collect adequate testing material, model studies were conducted consisting in the natural infestation, full chemical crop protection and inoculation with fungi from the genus Fusarium (F. culmorum and F. graminearum). The construction of a model based on a comprehensive statistical analysis may provide grounds for a description of susceptibility or resistance in individual wheat cultivars.

The aim of this study was to analyse the profile of fungal and plant metabolites, as well as antioxidant capacity of wheat cultivars. The analysis of ergosterol (ERG) as chemical fungal level indicator, mycotoxins from the group of trichothecenes and plant metabolites: 12 phenolic acids and free phenolic acids were conducted. 


\section{Materials and Methods}

\section{Plant material}

Twenty-three genotypes of winter wheat (Triticum aestivum L.) have been included in the study on the base of their resistance to Fusarium. A total of 13 wheat genotypes presented in Table $\mathrm{S} 1 *$ were provided by Polish plant breeding companies. Three lines were (82/2011, 83/2011 and 91/2011) selected at the Department of Genetics and Plant Breeding, Poznan University of Life Sciences. The other seven genotypes are cultivars and lines originated from Germany, the Czech Republic, Austria, Holland, Hungary, and Sweden, which are used in breeding of winter wheat as sources of resistance to Fusarium.

\section{Field trial}

The field experiment was randomized block system with 23 wheat genotypes and had 2 factors the variant of protection and the wheat genotype. All wheat genotypes were sown into $3 \mathrm{~m}^{2}$ plots in 3 replications in three different variants of protection:

1. natural infection (control) - (K);

2. chemical crop protection by fungicide application Duet Ultra $497 \mathrm{SC} 0.6$ 1/ha, and Capalo 337.5 SE 2 1/ha (OCH);

3. inoculation with fungi from the genus Fusarium (I).

The inoculum was produced from 6 Fusarium isolates exhibiting high pathogenicity, i.e. 3 isolates each obtained from $F$. graminearum and $F$. culmorum, with the isolates being preliminarily tested in field experiments. Spore concentration was established at approx. $10^{6}$ spores per $m l$. Next suspensions of all isolates were mixed.

Wheat heads were sprayed with the spore suspension at anthesis phonological stage at a rate of $100 \mathrm{ml} / \mathrm{m}^{2}$. Inoculations were performed individually on each plot at anthesis emergence, and repeated 3 days later at full anthesis stage. Plot disease severity for a population of approximately 200 spikes per plot was estimated for both incidence (percentage of infected spikes) and severity (percentage of infected spikelets of the diseased spikes). An FHB index (incidence $\times$ severity) $/ 100$ was calculated to assess plot severity (Groth et al. 1999).

From all genotypes $200 \mathrm{~g}$ bulk samples were collected, from which a subsample of $100 \mathrm{~g}$ grain was used in further analysis.

\section{Analytical methods}

Ergosterol was determined by high-performance liquid chromatography (HPLC). A detailed evaluation of the method was given in a study by Perkowski et al. (2008).

Grain samples were analysed for the presence of trichothecenes according to Perkowski et al. (2003).

\footnotetext{
*Further details about the Electronic Supplementary Material (ESM) can be found at the end of the article.
} 
For analysis of free phenolic acids samples of $50 \mathrm{~g}$ were collected. Samples were ground using a laboratory mill (WŻ-1) and phenolic compounds were extracted with $80 \%$ $\mathrm{MeOH}$. Samples of $10 \mathrm{~g}$ after flooding with $100 \mathrm{ml} \mathrm{MeOH}$ were placed in an ultrasound bath for $30 \mathrm{~min}$, then the precipitate was collected to distillation flasks and the extraction process was repeated three times. Next the combined extracts were evaporated to dryness in an evaporator. Phenolic compounds were transferred quantitatively to a vial using $\mathrm{MeOH}$ and dried in a stream of nitrogen. Total content of polyphenols was assayed according to Heimler et al. (2010). The amounts of $0.5 \mathrm{ml}$ of deionized water and $0.125 \mathrm{ml}$ of Folin-Ciocalteu reagent (Fluka) (Singleton and Rossi 1965) were added to $0.125 \mathrm{ml}$ of extract, and after 6 min the mixture was supplemented with $1.25 \mathrm{ml}$ of $7 \%$ aqueous $\mathrm{Na}_{2} \mathrm{CO}_{3}$ solution and $1 \mathrm{ml}$ of deionized water. After $90 \mathrm{~min}$, absorbance was read at a wavelength of $760 \mathrm{~nm}$ in relation to water (Helios spectrophotometer Thermo Electron Corp., MA, USA). Results were expressed in mg gallic acid/100 g d.m. sample.

Phenolic acid content was determined as described by Stuper-Szablewska et al. (2014). Briefly phenolic acids were assayed following basic and next acid hydrolyses. Phenolic acids were extracted from the inorganic phase using diethyl ether. Analysis of phenolic acid contents was conducted with a high performance liquid chromatograph (Waters SDS 501) coupled with a Waters 486 Tunable Absorbance Detector. Chromatographic separation was performed using a RP C18 column, $250 \times 4 \mathrm{~mm} \times 5 \mu \mathrm{m}$. A mixture of acetonitrile: $2 \%$ acetic acid in water was applied as the eluting phase $(\mathrm{pH}=2)$ (gradient). Concentrations of 12 phenolic acids were measured using the reference standard at the wavelengths of $320 \mathrm{~nm}$ and $280 \mathrm{~nm}$. The compounds were identified based on a comparison of retention times of the tested peaks with the retention times of the standard and by adding a specific amount of the standard to the tested samples, after which the analysis was repeated. The limit of detection was $1 \mu \mathrm{g} / \mathrm{g}$.

\section{Total antioxidant capacity}

The method for measuring the FRAP, i.e. "antioxidant power", was modified for grain samples according to Gliszczyńska-Świgło (2007).

\section{Statistical analysis}

Results recorded in the course of the conducted chemical analyses of twenty-three genotypes of winter wheat (Triticum aestivum L.) were subjected to statistical analysis using STATISTICA v 8.0 software. In order to compare contents of individual metabolites in samples, Tukey's multiple comparison procedure was used, with identical letters in rows or columns denoting a lack of differences at the significance level $\alpha=0.05$. Moreover, a stepwise linear discriminant analysis (SLDA) was performed in order to separate groups in the analysed populations and prepare a mathematical model (method used in statistics, pattern recognition and machine learning to find a linear combination of features that characterizes or separates two or more classes of objects or events). In this model Estimation of Discriminant Function Coefficients and determination the statistical significance 
and validity were prepared. The aim of discriminant analysis was to separate the experimental group (control, chemical protection and inoculation) based on the data of chemical analysis. The discriminant equation: $\mathrm{F}=\beta 0+\beta 1 \mathrm{X} 1+\beta 2 \mathrm{X} 2+\ldots+\beta \mathrm{pXp}+\varepsilon$, where, $\mathrm{F}$ is a latent variable formed by the linear combination of the dependent variable, $\mathrm{X} 1, \mathrm{X} 2, \ldots$ $\mathrm{Xp}$ are the $\mathrm{p}$ independent variables, $\varepsilon$ is the error term and $\beta 0, \beta 1, \beta 2, \ldots, \beta \mathrm{p}$ are the discriminant coefficients.

\section{Results}

The experimental model comprised three variants: natural infestation (K), natural infestation at the application of chemical crop protection $(\mathrm{OCH})$ and inoculation with Fusarium (I) in order to determine the effect of mass-scale infection on the development of fusarium head blight. Besides the traditional methods, the level of grain contamination was investigated using the analysis of ergosterol concentration (ERG). Moreover, the contents of mycotoxins from the group of trichothecenes were analysed quantitatively and qualitatively. Apart from these methods considered to be standard for such studies, a quantitative analysis was also conducted for 12 phenolic acids and free phenolic acids, while additionally the total antioxidant capacity was assessed. It was also decided to subject the recorded results to comprehensive statistical analysis in order to indicate the effect of inoculation on the concentration of produced metabolites and to determine their effect on the resistance response in plants.

Based on the results it was found that the fusarium head blight index (FHB index) ranged from $0 \%$ to $24 \%$ in the control, from $0 \%$ to $3 \%$ following chemical crop protection treatments and from $0 \%$ to $95 \%$ in case of inoculation (Table S1). Irrespective of the experimental variant, the lowest FHB index was recorded for genotypes Ertus, Praag 8, UNG 136.6.1.1, 20818 and 20816. Three of them are derived from genotype Sumai 3, a highly resistant spring wheat from China. Most registered Polish cultivars had low FHB index values at the application of chemical crop protection, while artificial inoculation caused a very high increase in the infestation rate, ranging from 50 to $95 \%$ FHB index.

The highest concentration of ergosterol was detected in seeds from inoculated plots, with a mean value of $26.69 \mathrm{mg} / \mathrm{kg}$ (Table 1). Approximately 3 times less ergosterol content was detected in the samples from control and chemically protected plants, with mean concentrations of this metabolite of $7.03 \mathrm{mg} / \mathrm{kg}$ and $9.93 \mathrm{mg} / \mathrm{kg}$. No significant differences were found between after the control and chemical crop protection treatments. However, highly significant differences were observed between the concentrations of this fungal metabolite after inoculation in relation to the two other groups. Similar differences between variants of the experiment were observed for the mean concentration of total trichothecenes. The highest mean concentration calculated for total toxin content was recorded at Fusarium inoculation treatment $(7.833 \mathrm{mg} / \mathrm{kg})$, while approx. 11 times lower amounts of mycotoxins were detected in the control and chemically protected plots (Table 1). There were significant differences in mean contents of DON and 3-AcDON between the inoculated group, and the control and the chemically protected group. In the case of the other toxins detected at slight concentrations, i.e. FUS-X, 15-AcDON and 


\begin{tabular}{|c|c|c|c|c|c|c|c|c|c|c|c|c|c|c|}
\hline 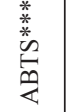 & 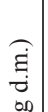 & $\begin{array}{l}\text { 苂 } \\
\text { ñ }\end{array}$ & $\begin{array}{l}\infty \\
\infty \\
\infty \\
+\end{array}$ & $\begin{array}{l}\stackrel{0}{.} \\
\stackrel{I}{J} \\
\end{array}$ & 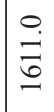 & 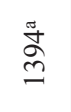 & $\hat{\dot{q}}$ & 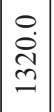 & \begin{tabular}{|c|}
0 \\
$\dot{a}$ \\
$\dot{\alpha}$ \\
- \\
\end{tabular} & 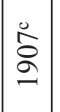 & 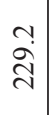 & 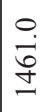 & $\begin{array}{l}0 \\
\infty \\
\stackrel{0}{\Delta} \\
\text { ते }\end{array}$ & \\
\hline 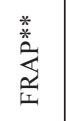 & 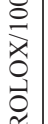 & $\underset{\stackrel{\sigma}{\sigma}}{\stackrel{\infty}{\sim}}$ & $\begin{array}{l}m \\
\infty \\
\infty \\
\sim\end{array}$ & $\begin{array}{l}0 \\
\hat{\dot{a}} \\
\stackrel{\vec{\lambda}}{ }\end{array}$ & 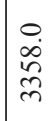 & 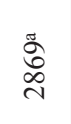 & $\begin{array}{l}\tilde{y} \\
\ddot{n} \\
\approx\end{array}$ & 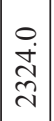 & $\begin{array}{l}0 \\
\dot{\leftarrow} \\
\dot{0} \\
+ \\
m\end{array}$ & $\left|\begin{array}{c}0 \\
n \\
2 \\
n \\
n\end{array}\right|$ & $\begin{array}{l}\infty \\
\dot{f} \\
\dot{f} \\
n\end{array}$ & $\mid \begin{array}{l}0 \\
\dot{0} \\
\infty \\
\infty \\
\sim\end{array}$ & $\begin{array}{l}\stackrel{0}{\grave{v}} \\
\hat{\sigma} \\
\sigma\end{array}$ & \\
\hline $\begin{array}{l}\text { 䓂 } \\
\text { 芒 }\end{array}$ & 惫 & $\begin{array}{l}\text { ळू } \\
\infty \\
\infty \\
\text { N }\end{array}$ & $\begin{array}{l}\frac{a}{\infty} \\
\vec{m}\end{array}$ & $\begin{array}{c}0 \\
\dot{v} \\
\dot{v} \\
\tilde{d}\end{array}$ & 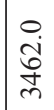 & $\begin{array}{l}\frac{\sigma}{\pi} \\
\stackrel{\infty}{\sim}\end{array}$ & $\begin{array}{l}\stackrel{0}{\mathrm{i}} \\
\stackrel{\tilde{n}}{ }\end{array}$ & 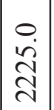 & $\begin{array}{c}0 \\
\dot{0} \\
\tilde{N} \\
n \\
m\end{array}$ & $\stackrel{ }{\underset{n}{n}}$ & $\begin{array}{l}\tilde{1} \\
\tilde{n} \\
\tilde{n}\end{array}$ & $\begin{array}{l}0 \\
0 \\
\stackrel{0}{2} \\
\stackrel{2}{2}\end{array}$ & 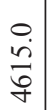 & \\
\hline 岕 & & 总 & $\overrightarrow{0}$ & $\begin{array}{l}0 \\
\dot{0} \\
i\end{array}$ & $\begin{array}{l}0 \\
\infty \\
\infty \\
\infty \\
+\end{array}$ & ले & $\begin{array}{l}\infty \\
\dot{q} \\
\end{array}$ & \begin{tabular}{|l|}
$\stackrel{0}{\vec{T}}$ \\
$\mathcal{F}$
\end{tabular} & $\begin{array}{l}0 \\
\infty \\
0 \\
0\end{array}$ & $\stackrel{\stackrel{ \pm}{7}}{\nabla}$ & $\overrightarrow{8}$ & 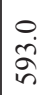 & $\begin{array}{l}\stackrel{\leftrightarrow}{1} \\
\infty \\
\infty\end{array}$ & \\
\hline 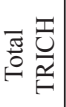 & & $\stackrel{\tilde{N}}{\tilde{F}}$ & 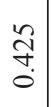 & $\mid \begin{array}{l}\infty \\
\stackrel{0}{0} \\
0\end{array}$ & \begin{tabular}{|l|}
$\vec{\infty}$ \\
$\stackrel{0}{0}$ \\
-
\end{tabular} & 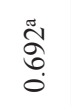 & $\begin{array}{l}\hat{a} \\
\tilde{c} \\
0\end{array}$ & $\begin{array}{c}0 \\
0 \\
0 \\
0\end{array}$ & ָे & $\begin{array}{l}\tilde{O} \\
\tilde{N} \\
\infty \\
\sim\end{array}$ & $\begin{array}{l}\stackrel{\sim}{\sim} \\
\stackrel{+}{f}\end{array}$ & 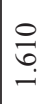 & $\begin{array}{l}\stackrel{0}{*} \\
\stackrel{\infty}{0}\end{array}$ & \\
\hline 妾 & & $\begin{array}{l}\ddot{\tilde{n}} \\
\stackrel{\infty}{0} \\
\dot{0}\end{array}$ & $\begin{array}{l}\stackrel{\infty}{\mathrm{N}} \\
\stackrel{0}{0}\end{array}$ & $\mid \begin{array}{l}\infty \\
0 \\
0 \\
0\end{array}$ & $\mid \begin{array}{l}0 \\
0 \\
0 \\
0\end{array}$ & 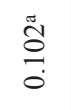 & $\underset{\stackrel{g}{I}}{\stackrel{0}{0}}$ & 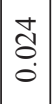 & $\begin{array}{l}n \\
\tilde{n} \\
0\end{array}$ & $\begin{array}{l}\tilde{a} \\
\ddot{0} \\
0 \\
0\end{array}$ & $\begin{array}{l}\stackrel{\infty}{=} \\
\vdots \\
0\end{array}$ & 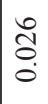 & $\begin{array}{l}\tilde{n} \\
\tilde{n} \\
0\end{array}$ & \\
\hline $\begin{array}{l}z \\
0 \\
0 \\
00 \\
\vdots \\
n \\
n\end{array}$ & $\stackrel{000}{*}$ & $\begin{array}{l}\frac{\pi}{\Delta} \\
\stackrel{0}{0}\end{array}$ & $\mid \begin{array}{l} \pm \\
0 \\
0 \\
0\end{array}$ & $\begin{array}{l}\infty \\
0 \\
0 \\
0\end{array}$ & $\begin{array}{l}\stackrel{a}{d} \\
\stackrel{0}{0}\end{array}$ & 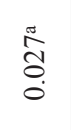 & $\begin{array}{l}0 \\
0 \\
0 \\
0\end{array}$ & $\begin{array}{l}0 \\
0 \\
0 \\
0\end{array}$ & $\begin{array}{l}0 \\
\dot{\theta} \\
\dot{0}\end{array}$ & 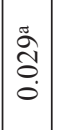 & $\begin{array}{l}\stackrel{1}{0} \\
\stackrel{0}{0}\end{array}$ & $\begin{array}{l}n \\
0 \\
0\end{array}$ & $\begin{array}{l}\hat{2} \\
0 \\
0 \\
0\end{array}$ & $\begin{array}{l}\text { 岁 } \\
\overrightarrow{0} \\
\underline{0}\end{array}$ \\
\hline $\begin{array}{l}z \\
0 \\
0 \\
0 \\
\vdots \\
j\end{array}$ & ह & $\begin{array}{l}\stackrel{a}{4} \\
\stackrel{2}{0} \\
\stackrel{0}{0}\end{array}$ & $\begin{array}{l}\tilde{\delta} \\
\tilde{0} \\
0\end{array}$ & $\begin{array}{l}8 \\
8 \\
0 \\
0\end{array}$ & $\mid \begin{array}{l}0 \\
\dot{0} \\
\dot{0}\end{array}$ & 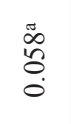 & $\begin{array}{l}0 \\
\tilde{\delta} \\
0 \\
0\end{array}$ & $\overline{8}$ & $\mid \begin{array}{l}\text { O } \\
0 \\
0\end{array}$ & $\begin{array}{l}\stackrel{0}{\exists} \\
\stackrel{+}{0}\end{array}$ & $\begin{array}{c}\stackrel{1}{\Xi} \\
\vdots \\
0\end{array}$ & $\begin{array}{l}m \\
0 \\
0 \\
0\end{array}$ & \begin{tabular}{l}
$\stackrel{n}{\infty}$ \\
\multirow{2}{*}{} \\
0
\end{tabular} & \\
\hline $\begin{array}{l}x \\
\dot{1} \\
\dot{D} \\
\text { D. }\end{array}$ & & $\begin{array}{l}\stackrel{\tilde{n}}{0} \\
\stackrel{0}{0}\end{array}$ & $\begin{array}{l} \pm \\
0 \\
0 \\
0\end{array}$ & $\begin{array}{l}8 \\
0 \\
0\end{array}$ & 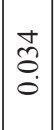 & $\begin{array}{l}\stackrel{\Xi}{\infty} \\
\stackrel{0}{0} \\
0\end{array}$ & $\begin{array}{l}m \\
0 \\
0 \\
0\end{array}$ & $\begin{array}{l}8 \\
0 \\
0 \\
0\end{array}$ & $\mid \begin{array}{l}\tilde{\sigma} \\
\tilde{\sigma} \\
0\end{array}$ & \begin{tabular}{|c|}
$\tilde{\infty}$ \\
$\tilde{I}$ \\
0 \\
$\dot{0}$
\end{tabular} & $\begin{array}{l}\tilde{0} \\
\dot{0} \\
0\end{array}$ & 吉 & $\begin{array}{l}0 \\
\tilde{\delta} \\
0 \\
0\end{array}$ & \\
\hline Z̆ & & $\begin{array}{l}\stackrel{5}{2} \\
\text { ñ } \\
0\end{array}$ & $\begin{array}{l}\hat{0} \\
\tilde{0} \\
0\end{array}$ & 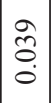 & 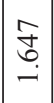 & $\begin{array}{l}\stackrel{a}{\infty} \\
\stackrel{\infty}{\infty} \\
0\end{array}$ & 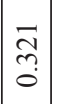 & $\begin{array}{l}0 \\
0 \\
0 \\
0\end{array}$ & $\mid$\begin{tabular}{l}
0 \\
0 \\
\hdashline \\
- \\
-
\end{tabular} & $\left|\begin{array}{l}0 \\
0 \\
n \\
n \\
r\end{array}\right|$ & $\begin{array}{l}\underset{T}{~} \\
\stackrel{+}{*}\end{array}$ & 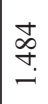 & $\begin{array}{l}\underset{N}{N} \\
\infty \\
\infty\end{array}$ & 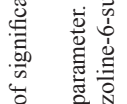 \\
\hline $\begin{array}{l}0 \\
\stackrel{x}{y}\end{array}$ & & $\underset{\sigma}{0}$ & $\mid \begin{array}{l} \pm \\
\sim \\
\infty \\
\dot{n} \\
\dot{n}\end{array}$ & $\begin{array}{l}0 \\
\infty \\
\\
-1\end{array}$ & $\mid \begin{array}{l}0 \\
\stackrel{2}{0} \\
\dot{0} \\
\dot{e}\end{array}$ & $\stackrel{\check{a}}{\alpha}$ & $\left|\begin{array}{l}2 \\
\infty \\
\dot{n} \\
\dot{n}\end{array}\right|$ & $\begin{array}{l}\infty \\
\infty \\
\infty \\
\dot{+}\end{array}$ & 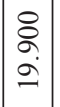 & 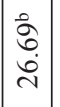 & 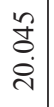 & $\begin{array}{l}0 \\
a \\
\dot{n} \\
\end{array}$ & 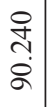 & . \\
\hline & & 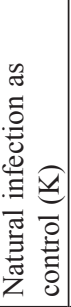 & 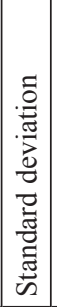 & $\begin{array}{l}\text { 音 } \\
\text { 㬅 } \\
\end{array}$ & 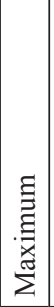 & 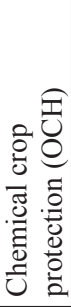 & 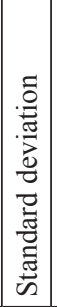 & 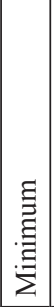 & 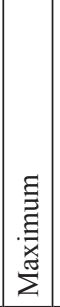 & 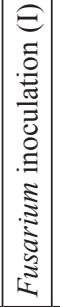 & 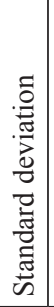 & 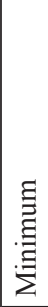 & 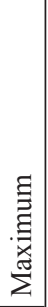 & $\tilde{d}_{0}^{*}$ \\
\hline
\end{tabular}




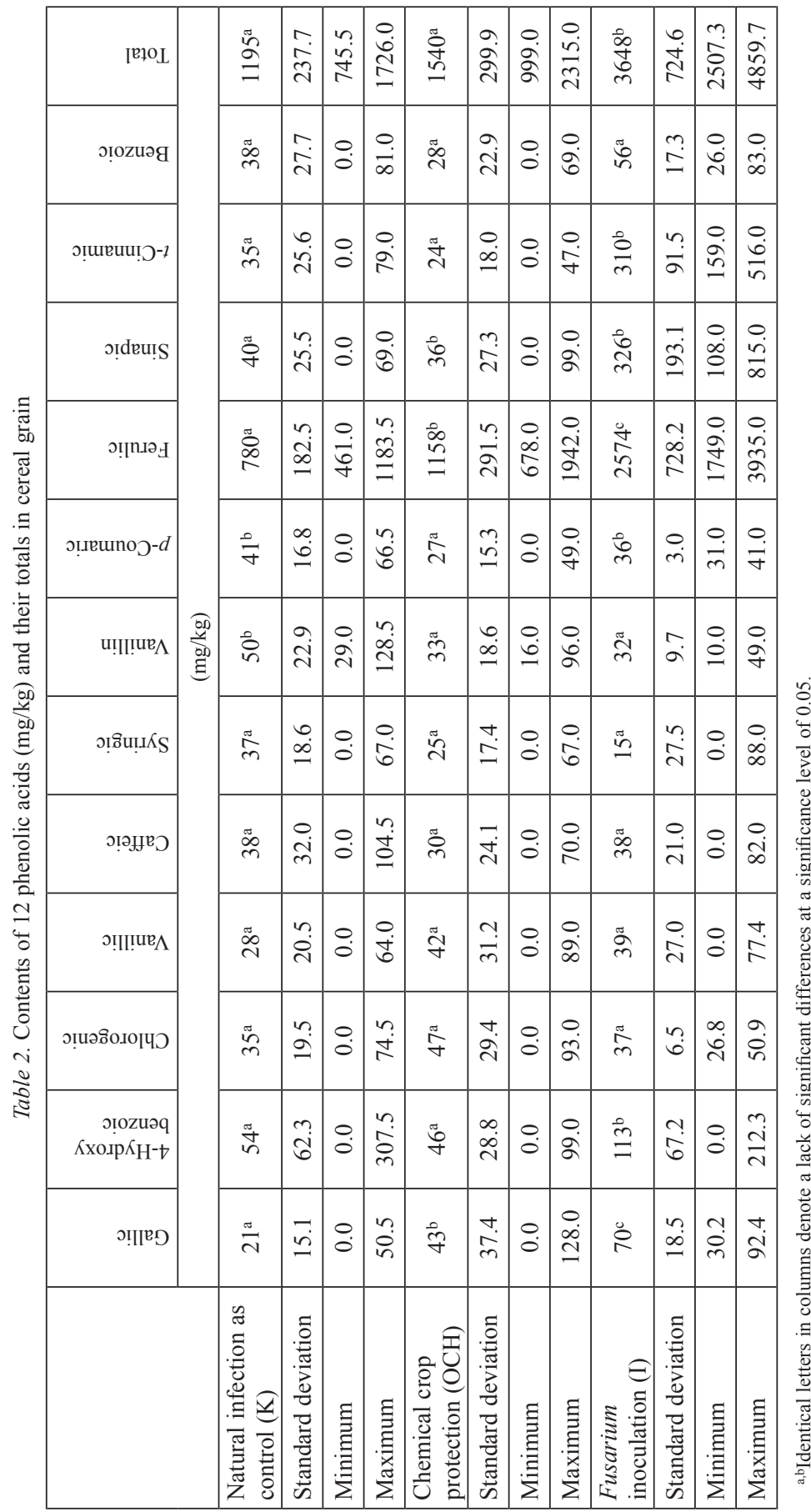




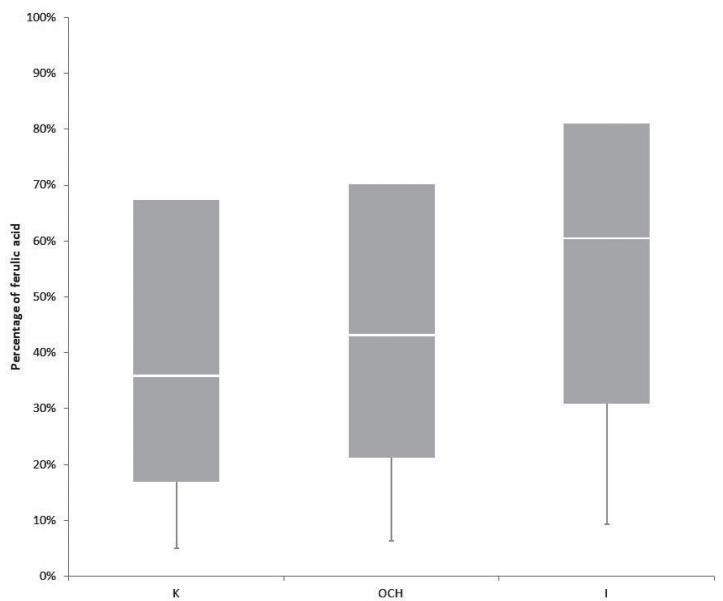

Figure 1. Percentage comparison of ferulic acid contents $(\mathrm{mg} / \mathrm{kg})$

NIV, no statistically significant differences were recorded between mean concentrations of these toxins.

Significant differences in the contents of individual phenolic acids between cultivars within each experimental variant were observed (Fig. S1). The highest concentrations were recorded for ferulic acid in all samples, the mean value for inoculated samples was $2574 \mathrm{mg} / \mathrm{kg}$, while for chemically protected samples it was lower $(1158 \mathrm{mg} / \mathrm{kg})$. The lowest concentration of this compound was recorded for the control $(780 \mathrm{mg} / \mathrm{kg})$.

In grain the content of ferulic acid was on average $377.8 \mu \mathrm{g} / \mathrm{g}$ for the control that proved lower in comparison to the chemical protection treatments (Table 2, Fig. 1). Con-
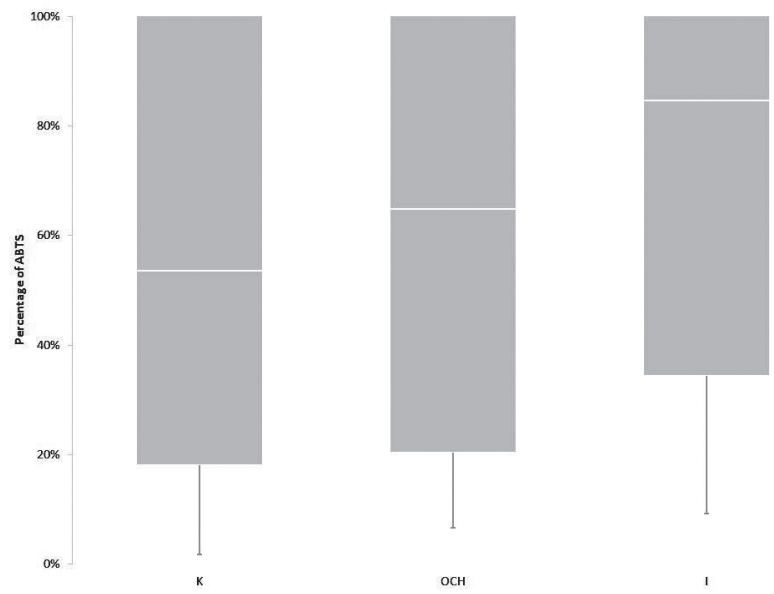

Figure 2. Percentage comparison of antioxidant capacity determined by ABTS ( $\mu$ mol TROLOX/100g d.m.) in the control, inoculated and chemically protected samples for 23 wheat genotypes 


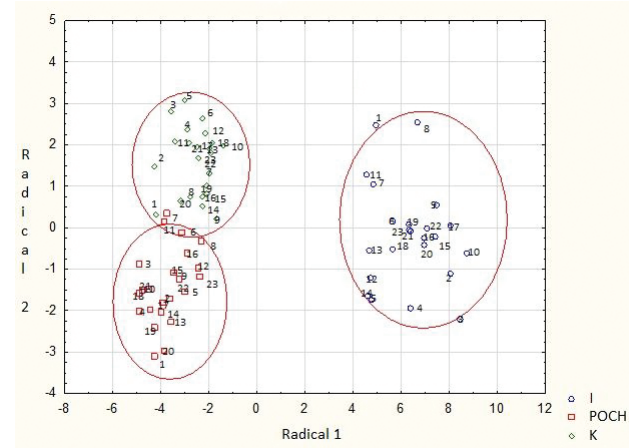

Figure 3. Canonical analysis of antioxidant capacity, free phenolic acids and phenolic acids in the control (K), chemically protected samples $(\mathrm{OCH})$ and inoculated (I). (gallic acid Wilk's lambda: 0.0246, 4-hydroxybenzoic acid Wilk's lambda: 0.0217, vanillic acid Wilk's lambda: 0.0205 , syringic acid Wilk's lambda: 0.0200, $t$-cinnamic acid Wilk's lambda: 0.0273 and benzoic acid Wilk's lambda: 0.0212)

tents of phenolic acids and ergosterol were also lower compared to the chemical protection treatment samples. Contents of ferulic acid, total phenolic acids and ergosterol (ERG) in grain for inoculation treatment increased in relation to chemically treated plants. Reaction to inoculation was related to genotypes of wheat and its resistance to infection by Fusarium. Many genotypes highly infected by Fusarium such as Muszelka, SMH and KBP. These lines were characterized by a very high increase of ferulic acid content after inoculation (Table S1). On the other hand, many resistant genotypes (Ertus, Fregate, UNG 136.6.1.1) reacted to a lower increase in the synthesis of ferulic acid after inoculation. Accordingly, the correlations for three and six genotypes with the largest and smallest FHB index were calculated (Table S2). In 3 genotypes with the highest FHB index the increase of ferulic acid was $326.6 \%$, and the total phenolic acids was $307 \%$; while in 3 the most resistant genotypes it was $121.7 \%$ for ferulic acid and $121.7 \%$ for total phenolic acids (Table S2).

Analyses of free phenolic acids (FPA) (Table 2) also showed significant differences in their contents between the tested populations of samples. The highest levels of free phenolic acids, similarly to bound phenolic acids, were recorded in samples of inoculated grain. This is probably due to the anti-stress effect caused in plants subjected to inoculation. In the case of antioxidant capacity analysed using three methods, its highest level was recorded in inoculated samples (I), while it was respectively lower for the control populations $\mathrm{K}$ and chemically protected populations OCH. Only ABTS showed statistically significant differences between all the groups (Fig. 2). The other methods differentiated significantly only groups $\mathrm{K}$ and $\mathrm{OCH}$ from group I (Table 2).

In all the experimental combinations, highly significant correlation was observed between contents of ferulic acid and total phenolic acids and between spike infestation parameters at $\alpha=0.05$.

Comparing acid profiles analysed, different behaviour was found to the stressor, which is the inoculation itself. In order to reflect changes in the content of individual fatty acids 
mathematical operation was performed. This operation involved the subtraction of the average content of each fatty acid in control and inoculated samples. On the basis of the resulting graph (Fig. 3) showed that the phenolic acids, with the exception of the new syringic acid, vanillin and $p$-coumaric are produced by the plant when fungal infection in a higher concentration than in its growth-free natural stressor (differential negative) as proposed by Wojcieszyńska et al. (2013). Caffeic acid concentration in plant tissues was not affected by infection.

In order to investigate the interrelations between results of chemical assays, they were analysed statistically to construct a mathematical model in order to determine which of the investigated and assayed chemical compounds have the greatest discrimination power resulting in the separation of the three analysed populations: $\mathrm{K}$, I and $\mathrm{OCH}$. The assumed mathematical model was based on the step-wise discrimination analysis. The model was supplemented successively with all the metabolites. Firstly the level of contamination with fungi (ERG) and mycotoxins (concentration of trichothecenes) was analysed. Phenolic acids, free phenolic acids and antioxidant capacity were combined in the canonical analysis (Fig. 2). The obtained separation of the analysed genotypes between the tested populations was high; for samples I it was $100 \%$, for $\mathrm{OCH}$ it was $95 \%$, and for $\mathrm{K}$ it was $90 \%$. Amongst 12 analyzed phenolic acids the highest discrimination power was found for gallic acid, 4-hydroxybenzoic acid and vanillic acid (Fig. 3).

\section{Discussion}

The mean concentration of group B trichothecenes and some phenolic acids, including ferulic acid was higher after $\mathrm{OCH}$ treatment than in natural infection. It is very difficult to refer to this fact in view of the results collected so far. The observed differences between contents of fungal metabolites and phenolic acids between the population of $\mathrm{K}$ and $\mathrm{OCH}$ samples may be explained by the fact that sublethal concentrations of fungicides trigger trichothecene biosynthesis (Ochiai et al. 2007; Müllenborn et al. 2008). On the other hand, all fungicides (prothioconazole, tebuconazole, metconazole and prothioconazole + tebuconazole) used in the study reduced FHB index and deoxynivalenol (DON) levels according to Amarasinghe et al. (2013).

Some authors stated that although some of the fungicides are ineffective against FHB, some have been shown to stimulate DON and NIV production particularly at sub-optimal fungal growth conditions and low fungicide dosage (Jennings et al. 2000; Magan et al. 2002). Magan et al. (2002) suggested that environmental stress factors, particularly water availability and temperature, and low fungicide doses, may stimulate mycotoxin production by fusaria in vitro and in wheat grain. Mesterhazy et al. (2003) found that fungicide efficacy in controlling FHB and DON accumulation was greater in more resistant than in more susceptible winter wheat cultivars.

Resistance mechanisms in plants may be influenced by different factors connected with plant immunity. In this respect the greatest role is played by antioxidant compounds. Other authors also indicated the resistance aspect when stressing the role of phenolic compounds in pathogenesis (Lattanzio et al. 2006). Among these compounds we need to 
focus on ferulic acid, which is found in cereals at the highest concentration. Based on these observations it was found that phenols have the most significant effect on defence mechanisms in plants against induced stress occurring during inoculation or chemical crop protection treatment.

Ferulic acid is a component of non-enzymatic resistance mechanism of the plant. Use of two stressors which are inoculation and chemical protection caused the plant reaction to stress factors - higher synthesis of ferulic acid. There were not stressors during the growing season in the control plots so the plants did not produce big amounts of ferulic acid.

Considerable differences in contents of ferulic acid, observed in the conducted experiment, may be reflected in genotype differences in resistance to Fusarium. Siranidou et al. (2002) found that contents of ferulic acid in glumes of cv. 'Frontana' resistant to Fusarium was over 2 -fold higher than in the sensitive cv. 'Agent'. These authors stated than definitely phenolic acids play a role in the resistance of cv. 'Frontana' to F. culmorum. In our study an increase of ferulic acid synthesis after inoculation, compared to control was related to genotypes of wheat. Increase in ferulic acid between inoculation and control was more strongly associated with genotypic differences than just the amount of ferulic acid in grains. Bily et al. (2003) stated that ferulic acid influences the pathway of synthesis for mycotoxins produced by Fusarium. It was concluded that ferulic acid in resistance reactions of wheat to infections caused by Fusarium sp. influences the amount of Fusarium toxins produced in grain (Engelhardt et al. 2006). Numerous in vitro experiments confirmed the inhibitory effect of selected phenolic acids on the production of mycotoxins produced, e.g. by Fusarium culmorum or Fusarium verticillioides (Beekrum et al. 2003; Boutigny et al. 2010).

FHB index is the highest in inoculation treatment, lower in control treatment and the lowest in $\mathrm{OCH}$ treatment due to chemical protection of that plots. Reaction to inoculation was related to genotypes of wheat and its resistance to infection by Fusarium. Phenolic acids, with the exception of the new syryngic acid, vanillin and $p$-coumaric are produced by the plant in bigger amount after fungal infection. Amongst 12 analysed phenolic acids the highest discrimination power was found for gallic acid, 4-hydroxybenzoic acid and vanillic acid. Based on the results it was found that the use of two stressors which are inoculation and chemical protection caused the plant reaction - higher synthesis of group B trichothecenes and some phenolic acids, including ferulic acid. Results of this study indicate a significant effect of mass infection with fungi from the genus Fusarium not only on the content of ferulic acid, but also other derivatives of cinnamic acid.

\section{Acknowledgement}

This work was supported by the National Science Centre in Poland (project no. NCN 2012/07/B/NZ9/02385). 


\section{References}

Amarasinghe, Ch.C., Tamburic-Ilincic, L., Gilbert. J,, Brûlé-Babel, A.L., Fernando, W.G.D. 2013. Evaluation of different fungicides for control of fusarium head blight in wheat inoculated with 3ADON and 15ADON chemotypes of Fusarium graminearum in Canada. Can. J. Plant Pathol. 35:1-9.

Bartók, T., Lamper, C. 2003. Influence of cultivar resistance, epidemic severity, and Fusarium species on the efficacy of fungicide control of Fusarium head blight in wheat and deoxynivalenol (DON) contamination of grain. Plant Dis. 87:1107-1115.

Beekrum, S., Govinden, R., Padayachee, T., Odhav, B. 2003. Naturally occurring phenols: a detoxification strategy for fumonisin B1. Food Addit. Contam. 20:490-493.

Bily, A.C., Reid, L.M., Taylor, J.H. 2003. Dehydrodimers of ferulic acid in maize grain pericarp and aleurone: resistance factors to Fusarium graminearum. Phytopathol. 93:712-719.

Bottalico, A. 1998. Fusarium diseases of cereals: species complex and related mycotoxin profiles in Europe. J. Plant Path. 80:85-103.

Boutigny, A.L., Richard-Forget, F., Barreau, C. 2008. Natural mechanisms for cereal resistance to the accumulation of Fusarium trichothecenes. Eur. J. Plant Path. 121:411-423.

Boutigny, A.L., Atanasova-Pénichon, V., Benet, M., Barreau, C., Richard-Forget, F. 2010. Natural phenolic acids from wheat bran inhibit Fusarium culmorum trichothecene biosynthesis in vitro by repressing Tri gene expression. Eur. J. Plant Pathol. 127:275-286.

Chrpová, J. Šíp, V., Štočková, L., Milec, Z. Bobková, L. 2010. Resistance of winter wheat varieties registered in the Czech Republic to Fusarium head blight in relation to the presence of specific Rht alleles. Czech J. Genet. Plant Breeding 46:122-134.

Colla, P., Gilardi, G., Gullino, M.L. 2012. A review and critical analysis of the European situation of soilborne disease management in the vegetable sector. Phytoparasitica 40:515-523.

Engelhardt, G., Barthel, J., Sparrer, D. 2006. Fusarium mycotoxins and ochratoxin A in cereals and cereal products: results from the Bavarian Health and Food Safety Authority in 2004. Mol. Nutr. Food Res. 50:401-405.

Gliszczyńska-Świgło, A. 2007. Folates as antioxidants. Food Chem. 101:1497-1500.

Groth, J.V., Ozmon, E.A., Busch, R.H. 1999. Repeatability and relationship of incidence and severity measures of scab of wheat caused by Fusarium graminearum in inoculated nurseries. Plant Dis. 83:1033-1038.

Heimler, D., Vignolini, P., Isolani, L., Arfaioli, P., Ghiselli, L., Romani, A. 2010. Polyphenol content of modern and old varieties of Triticum aestivum L. and T. durum Desf. grains in two years of production. J. Agric. Food Chem. 58:7329-7334.

Homdork, S., Fehrmann, H., Beck, R. 2000. Effects of field application of tebuconazole on yield, yield components and the mycotoxin content of fusarium-infected wheat grain. J. Phytopathol. 148:1-6.

Jennings, P., Turner, J.A., Nicholson, P. 2000. Overview of Fusarium ear blight in the UK - effect of fungicide treatment on disease control and mycotoxin production. In: Proc. of the BCPC Conf. 2000 - Pests and Diseases. British Crop Protection Council. Farnham, UK. 2:707-712.

Kubo, K., Kawada, N., Nakajima, T., Hirayae, K., Fujita, M. 2014. Field evaluation of resistance to grain infection and mycotoxin accumulation caused by Fusarium head blight in western Japanese wheat (Triticum aestivum L.) cultivars. Euphytica 200:81-93.

Lattanzio, V., Lattanzio, V.M.T., Cardinali, A. 2006. Role of phenolics in the resistance mechanisms of plant against fungal pathogens and insects. Phytochemistry: Advances in Research. Research Signpost. Kerala, India. pp. 23-67.

Magan, N., Hope, R., Colleate, A., Baxter, E.S. 2002. Relationship between growth and mycotoxin production by Fusarium species, biocides and environment. Eur. J. Plant Path. 108:685-690.

Mesterhazy, A., Bartok, T., Lamper, C., 2003. Influence of wheat cultivar, species of Fusarium, and isolate aggressiveness on the efficacy of fungicides for control of Fusarium head blight. Plant Disease 87:11071115 .

Müllenborn, C., Steiner, U., Ludwig, M., Oerke, E.Ch. 2008. Effect of fungicides on the complex of Fusarium species and saprophytic fungi colonizing wheat kernels. Eur. J. Plant Path. 120:157-166. 
Ochiai, N., Tokai, T., Takahashi-Ando, N., Fujimura, M., Kimura, M. 2007. Genetically engineered Fusarium as a tool to evaluate the effects of environmental factors on initiation of trichothecene biosynthesis. FEMS Microbiol. Letters 275:53-61.

Paterson, R.R.M., Lima, N. 2010. How will climate change affect mycotoxins in food? Food Res. Int. 43:19021914.

Perkowski, J. 1999. Badania zawartości toksyn fuzaryjnych w ziarnie zbóż (Analyses of fusarium toxin contents in cereal grain). Rocz. Akademii Roln. W Poznaniu, Rozprawy Naukowe, Book 295. Poznan, Poland. 136 p. (in Polish)

Perkowski, J., Buśko, M., Stuper, K., Kostecki, M., Matysiak, A., Szwajkowska-Michałek, L. 2008. Concentration of ergosterol in small-grained naturally contaminated and inoculated cereals. Biologia 63:542-547.

Perkowski, J., Kiecana, I., Kaczmarek, Z. 2003. Natural occurrence and distribution of fusarium toxins in contaminated barley cultivars. Eur. J. Plant Path. 109:331-339.

Schaafsma, A.W., Hooker, D.C. 2007. Climate models to predict occurrence of Fusarium toxins in wheat and maize. Int. J. Food Microbiol. 119:116-125.

Singleton, V.L., Rossi, J.A. 1965. Colorimetry of total phenolics with phosphomolybdic-phosphotungstic acid reagents. Am. J. Enol. Vitic. 16:144-158.

Siranidou, E., Kang, Z., Buchenauer, H. 2002. Studies on symptom development, phenolic compounds and morphological defence responses in wheat cultivars differing in resistance to fusarium head blight. J. Phytopathol. 150:200-208.

Stuper-Szablewska, K., Kurasiak-Popowska, D., Nawracała, J., Perkowski, J. 2014. Comparison of phenolic acid contents in various wheat genotypes. Przem. Chem. 93:2274-2278.

Wojcieszynska, D., Hupert-Kocurek, K., Guzik, U. 2013. Mikrobiologiczny rozkład kwasu cynamonowego i jego hydroksypochodnych (Microbiological degradation of cinnamic acid and its hydroxyl-derivatives). Postep. Mikrobiol. 52:135-141. (in Polish)

\section{Electronic Supplementary Material (ESM)}

Electronic Supplementary Material (ESM) associated with this article can be found at the website of CRC at http://www.akademiai.com/content/120427/

Electronic Supplementary Table S1. Fusarium head blight index and ferulic acid content

Electronic Supplementary Table S2. Contents of ferulic acid. total phenolic acids and ergosterol (ERG) in grain of 3 and 6 genotypes with the highest and 3 and 6 genotypes with the lowest fusarium head blight index depending on the experimental variant

Electronic Supplementary Figure S1. Differences in concentrations of analysed phenolic acids between control and inoculation in terms of their order in the mechanism of biosynthesis 